\title{
Neural Network Based on Orthogonal Polynomials Applied in Magnetic Levitation System Control
}

\author{
Miroslav B. Milovanovic ${ }^{1}$, Dragan S. Antic ${ }^{1}$, Sasa S. Nikolic ${ }^{1}$, Stanisa Lj. Peric ${ }^{1}$, \\ Marko T. Milojkovic ${ }^{1}$, Miodrag D. Spasic ${ }^{1}$ \\ ${ }^{1}$ University of Nis, Faculty of Electronic Engineering, Department of Control Systems, \\ Aleksandra Medvedeva 14, Nis, Republic of Serbia \\ sasa.s.nikolic@elfak.ni.ac.rs
}

\begin{abstract}
This paper presents a new approach for improving performances of magnetic levitation system. Controlled parameter is the amplitude which levitated object achieves during movement from one levitation position to another. Two position levitation with improved amplitude performances is obtained by implementing orthogonal neural network in standard levitation control logic. Proposed network is a nonlinear autoregressive neural network with newly developed activation function based on orthogonal polynomials. Performed experiments on a system with default control logic showed that it could not provide stable two position levitation when specified amplitude of the levitation object is greater than $10^{-4} \mathrm{~m}$. Artificial network was trained using real experimental data and it was based on standard tangent and sigmoid activation functions. Default activation functions were then substituted with a newly developed orthogonal polynomial functions. The amplitude $10^{-3} \mathrm{~m}$ was achieved with stable two position levitation after parameter optimization. It is proven that simple control logic with nonlinear autoregressive neural network and proper activation function can provide improved amplitude performances.
\end{abstract}

Index Terms-Activation function; magnetic levitation system; neural networks; orthogonal polynomials.

\section{INTRODUCTION}

The main focus of this paper is the improvement of two position levitation performances of magnetic levitation system (MLS). Different methods for establishing stable levitation performances are already presented in many existing papers. Two position control scheme based on the lead-lag control and the sliding mode control (SMC) of a stage system is used in [1]. Another usage of SMC for the purpose of levitation control is presented in [2]. Linear and nonlinear controllers for controlling the height of a steel ball during the levitation process are presented in [3]. Position control of a MLS is achieved using a Lyapunov function [4]. A generalized proportional integral controller, which compensates the errors during levitation is shown in [5]. The controller provides robustness of a closed-loop system and shows satisfactory experimental results for stabilization and

Manuscript received 14 June, 2016; accepted 22 December, 2016.

This paper was realized as a part of the projects TR 35005, III 43007 and III 44006, financed by the Ministry of Education and Science of the Republic of Serbia for the period 2011-2016. trajectory tracking tasks. Fuzzy-Tuning PID controller which uses fuzzy logic to adjust PID parameters is proposed in [6]. Intelligently adjusted controller showed the improvement of performances and greater flexibility than a conventional PID controller. Other examples of fuzzy logic usage for the purpose of levitation control are shown in [7-10]. Next widely used technique for position control of a levitation object in MLS is model predictive control (MPC). The simulation results and obtained levitation performances by using MPC are presented in [11], [12].

In this paper, a laboratory model of MLS, manufactured by Inteco [13], is used for experimental purposes. The main goal of the experiments was to improve two position levitation performances of the metal ball by implementing improved neural network in the system control logic. The network implementation in a control logic introduces a more efficient control approach in control systems theory. Usage of a feedforward network with one hidden layer in levitation control logic is presented in [14]. An intelligent method is proposed for controlling three degrees of freedom of magnetic suspension system in [15]. Educational paper [16] describes a real-time digital control environment with a magnetic levitation device usage, optimized for neural network implementation. Reference control model of the levitation system is a neural network in [17] where the embedded linear model is used for network weights forming procedure. A hybrid model of a neural network, which is based on a radial basis function, is designed for modelling a MLS in [18]. The model is capable to control the levitation process and to provide precisely tracking of reference signal. Finally, it can be concluded from [1]-[18] that systems which include soft computing techniques inside standard control logics could possess better control performances compared to conventional controllers.

Herein, a maximal distance (amplitude) from the first levitation position to the second levitation position, during two position levitation process, will be used as the performance parameter. The levitation system with default linear quadratic (LQ) tracking mode control logic will be used for experimental purposes. Finally, tracking control logic will be modified by a neural network implementation and three different control logics will be experimentally 
tested. Disadvantages of examined controls will be observed and improved performances obtained by network implementation will be presented.

\section{LQ TRACKING MODE CONTROL LOGIC AND NEURAL NETWORK IMPLEMENTATION}

Standard linear quadratic (LQ) tracking mode control logic is used to achieve two position state of the levitating object. LQ controller can be used when the state of the plant is available for direct measurement. It is often used for tracking specified trajectories, which will be of main interest for this paper. Also, it is very effective for stabilization of the closed loop system. LQ control is based on minimization of a quadratic cost function. The feedback control law of LQ controller can be expressed as

$$
u(t)=-K x(t)=\left(-R^{-1} B^{T} P(t)\right) x(t),
$$

where $R$ is a matrix which defines the control input weights in the cost function, $B$ is an input matrix in the state space model, $P(t)$ is the solution of a Riccati differential equation. Generally, $K$ represents a gain of a state feedback which is time dependent. In this paper, LQ controller operates in the way that the velocity of the suspended body, coil current values and the control signal are recalculated for each new value of the ball position. The suspended body of this magnetic levitator is a metallic ball of $57.1 \mathrm{~g}$ mass. Block diagram of the system with LQ tracking mode control logic is shown in Fig. 1. Additional information could be found in [13].

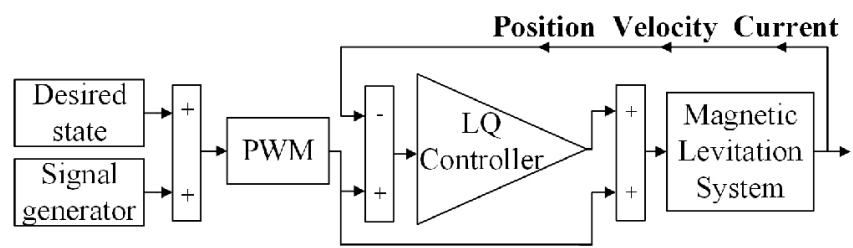

Fig. 1. Block diagram of LQ tracking mode control system.

The signal generator block and the Pulse Width Modulation (PWM) block in Fig. 1 are the most important parts of the input signal forming procedure. The signal generator block can produce one of three different waveforms: sine wave, square wave and sawtooth wave. Amplitude and frequency parameters determine the values of the desired output signal. Square wave signal will be used in the experiments. The PWM block generates excitation pulses. The signal generator time period is adjusted to $T=$ $4 \mathrm{~s}$ and two different movement amplitudes for generating desired square wave signal are selected $\left(A_{1}=10^{-4} \mathrm{~m}\right.$ and $A_{2}=10^{-3} \mathrm{~m}$ ).

Next step was to implement a neural network inside control logic from Fig. 1. Block diagram of the levitation system with an integrated neural network is shown in Fig. 2.

The network is implemented in the system to improve the characteristics of the input signal. The sum of the LQ controller output signal and the network output signal is formed, and its mean value is calculated as
$U_{M L}=\frac{U_{L Q}+U_{N N}}{2}$. Variable $U_{M L}$ is a final control signal which is forwarded to the MLS input, $U_{L Q}$ is a control signal on the LQ controller output and $U_{N N}$ is a neural network control signal.

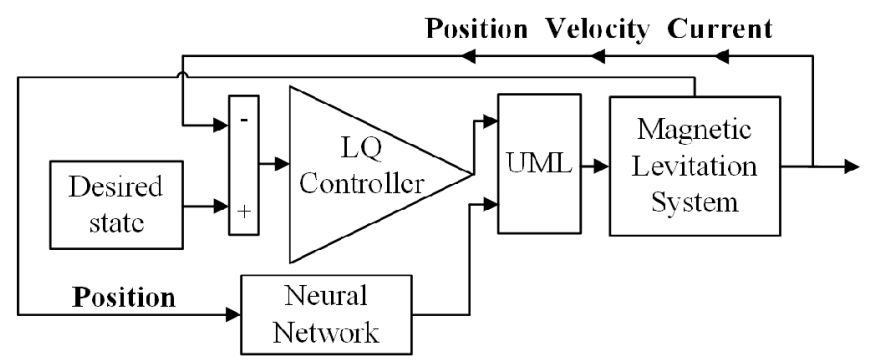

Fig. 2. Block diagram of control system with integrated neural network.

Nonlinear autoregressive neural network with external inputs (NARX) [19] was used as a foundation in this paper. It was shown in our previous researches that this type of the network is an optimal solution for implementation inside Inteco MLS control logics [13], [19], [20].

Two types of experiments are performed on the system which control logics are presented in Fig. 1 and Fig. 2. Three different control logics are used for each experiment. First one is the default LQ control logic (Fig. 1). Second and third control logics are based on modified logics from Fig. 2. The difference is the type of implemented activation function inside the network. Smooth functions are used for obtaining better stability performances because they could smooth out local minima of the total output of the network. Sigmoid activation function (2) is used in the first modified logic, and hyperbolic tangent function (3) is implemented in the second one. Both activation functions are described in [21]:

$$
\begin{gathered}
y(x)=\frac{1}{1+e^{-x}}, \\
y(x)=\frac{2}{1+e^{-2 x}}-1 .
\end{gathered}
$$

The network was trained with real experimental data obtained from levitation system. Current values from electromagnetic coil are chosen for input data. Position values which metallic ball had at individual moments of the time are used for network outputs. Input and output signals were sampled every $1 \mathrm{~ms}$ for a time period of $20 \mathrm{~s}$. On the basis of this, resulting input and output training vectors had 20000 samples each. Training method is selected by the empirical testing procedure. The neural network is trained with commonly used training types which are implemented inside Matlab software. The best performances (the least mean squared errors) are obtained when basic Quasi-Newton method is used. Quasi-Newton method is unconstrained optimization algorithm which possesses fast convergence. One iteration of updating neural network weights during the training procedure, by using this method, can be presented as

$$
\omega_{i}^{k+1}=\omega_{i}^{k}+\alpha^{k} d^{k}
$$


where $d^{k}$ is the direction parameter which purpose is to approximate Newton's direction, $\alpha^{k}$ is the step size, $\omega_{i}^{k}$ and $\omega_{i}^{k+1}$ are values of weight coefficient $\omega_{i}$ at iterations $k$ and $k+1$, respectively. Direction parameter $d^{k}$ can be obtained from

$$
d^{k}=-B^{k} \nabla f\left(\omega^{k}\right)
$$

where $B^{k}$ is a positive definite matrix which is adjusted from iteration to iteration.

Fifteen neurons are used in the hidden layer of the neural network. Time period $T=4 \mathrm{~s}$ and amplitude value $A_{1}=10^{-4} \mathrm{~m}$ are used for setting up the first experiment. Specified amplitude presents desired movement (distance from the first levitation position to the second levitation position). Experimental results of the ball levitation process (during the time interval $t=10 \mathrm{~s}$ ) are shown in Fig. 3.

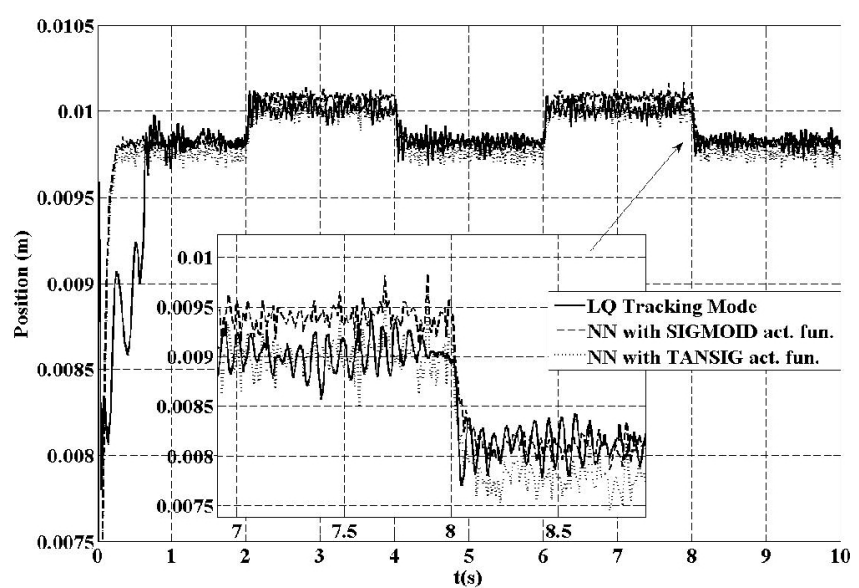

Fig. 3. Comparison of two-position ball movement for three different control logics, for specified amplitude $A_{1}=10^{-4} \mathrm{~m}$.

It can be concluded that two positional state of the ball is successfully achieved with all three control logics. Oscillations in all cases are up to $1 \%$ compared to the desired position, which are satisfactory results. Irregularities during the first second are excluded because they are the consequences of a human factor (the ball placement in levitation position is performed manually).

Identical control logics are used for the second experiment. Amplitude value is the only change which is made. The task was to test the system with larger movement which the ball must perform during position switching. Amplitude value is set to $A_{2}=10^{-3} \mathrm{~m}$, which increased desired movement 10 times compared to the first experiment ( $\left.A_{2} / A_{1}=10\right)$. Poor position performances after increasing specified amplitude can be seen in Fig. 4. The system is losing control of suspended ball for all three control logics after first time period $T=4 \mathrm{~s}$. Lost control caused the levitation process to interrupt (position approximately equal to 0.014 in Fig. 4). It is also obvious from Fig. 4 that presence of oscillations is significantly greater compared to results from the first experiment. The system is not capable to establish control of the ball again itself, so performed an experiment failed to complete.

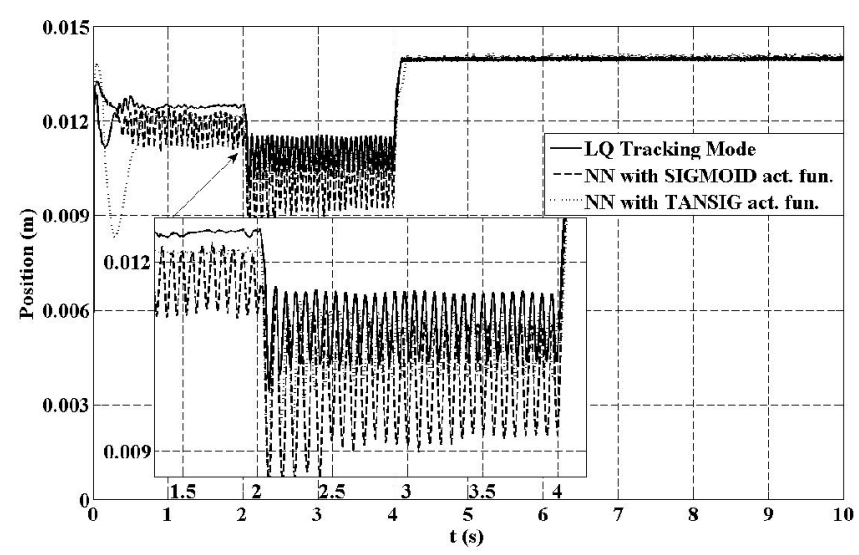

Fig. 4. Comparison of two-position ball movement for three different control logics, for specified amplitude $A_{2}=10^{-3} \mathrm{~m}$.

Two experiments are repeated multiple times and the results changes were negligible. It can be concluded that default and modified control logics can satisfactory control the ball during the levitation process when the specified amplitude is the most $10^{-4} \mathrm{~m}$. Poor control performances and unsatisfactory results are obtained for all three control logics by selecting levitation amplitude to be $10^{-3} \mathrm{~m}$. A newly developed neural network based on orthogonal activation functions will be presented in the next section with purpose to improve these poor performances.

\section{NEURAL NETWORK ACTIVATION FUNCTIONS BASED ON ORTHOGONAL POLYNOMIALS}

Reasons for using orthogonal functions inside neural networks are stability and tracking performances improvement - which these functions could provide to a system [22]. In this paper, activation functions from Section II will be changed with the new orthogonal trigonometric function [23]

$$
\varphi(t)=e^{-t} \sin t
$$

The complete necessary mathematical background of this orthogonal trigonometric polynomials can be found in [18]. The newly obtained generalized quasi-orthogonal polynomials of order $k=1$ [20] were used for experimental purposes. These polynomials are a generalization of previously performed quasi-orthogonal polynomials, also carried out by the authors of this paper [24]. The usage of these polynomials is justified by two reasons: considered MLS is a technically imperfect system [25]-[27], and second, orthogonal polynomials can have stabilization function when they are used as neural network activation functions [20]. Mathematical apparatus for performing these polynomials will be briefly presented below. Generalized polynomials of Legendre type are combination of three polynomial classes (very suitable for further considerations), almost [25], [26], improved almost [27] and quasiorthogonal Legendre polynomials [20]. The can be represented by 


$$
P_{n}^{(k, \delta)}(x)=\sum_{i=0}^{n} A_{n, i}^{(k, \delta)} x^{i},
$$

where

$$
A_{n, i}^{(k, \delta)}=(-1)^{n+i+k} \frac{\prod_{j=1}^{n-k}(i+j \delta)}{i !(n-i) !} .
$$

In (7) and (8) $k$ represents a quasi-orthogonal order, whereas $\delta$ is a constant, defined as [20], [25]

$$
\delta=1+\varepsilon, \quad 0<\varepsilon<<1 .
$$

Parameters $\delta$ and $\varepsilon$ are uncertain quantities, which describe imperfection of the system. Variations of $\delta$ and $\varepsilon$ contain cumulative impacts of all imperfect elements, measurement noise on the system output, and model uncertainties. The responses are in certain boundaries, which depend mostly on parameters $\delta$ and $\varepsilon$. Boundaries could be also dependent on the system components quality, and the noise level which is present in a signal. Inner product orthogonality of the interval $(0,1)$ with weight $w(x)=1$ is

$$
\int_{0}^{1} P_{m}^{(k, \delta)}(x) P_{n}^{(k, \delta)}(x) d x= \begin{cases}Q_{k}(\delta), & m \neq n, \\ N_{n, n}^{k, \delta}, & m=n,\end{cases}
$$

where $Q_{k}(\delta)=q_{0}+q_{1} \delta+q_{2} \delta^{2}+\cdots+q_{k} \delta^{k}$ represents the polynomial, which for a given value of the $\delta$ has a finite value. Several generalized first order quasi-orthogonal polynomials $(k=1)$ on the interval $(0,1)$ with weight coefficient $w(x)=1$ are given by:

$$
\left\{\begin{array}{l}
P_{1}^{(1, \delta)}(x)=a_{11}^{(1, \delta)} x+a_{10}^{(1, \delta)} \\
P_{2}^{(1, \delta)}(x)=a_{22}^{(1, \delta)} x^{2}+a_{21}^{(1, \delta)} x+a_{20}^{(1, \delta)} \\
P_{3}^{(1, \delta)}(x)=a_{33}^{(1, \delta)} x^{3}+a_{32}^{(1, \delta)} x^{2}+a_{31}^{(1, \delta)} x+a_{30}^{(1, \delta)} \\
\vdots
\end{array}\right.
$$

where $a_{11}^{(1, \delta)}=-1, a_{10}^{(1, \delta)}=1, a_{22}^{(1, \delta)}=-\frac{(\delta+2)}{2}$,

$a_{21}^{(1, \delta)}=(\delta+1), a_{21}^{(1, \delta)}=(\delta+1), a_{20}^{(1, \delta)}=-\frac{\delta}{2}$,

$a_{33}^{(1, \delta)}=-\frac{(\delta+3)(2 \delta+3)}{6}, a_{32}^{(1, \delta)}=(\delta+1)(\delta+2)$,

$a_{31}^{(1, \delta)}=-\frac{(\delta+1)(2 \delta+1)}{2}, a_{30}^{(1, \delta)}=\frac{\delta^{2}}{3}$.

\section{Newly DeVeloped Neural NeTwork AND EXPERIMENTAL RESULTS}

Experimental results of applied generalized quasiorthogonal polynomials (11) are presented in Table I. Testing procedure is performed by selecting values of the parameter $\delta$ from the range 1.000-1.050 with step size 0.005, according to (9). Levitation experiments are done for functions (11), and obtained results are given in Table I. Four levitation states are experimentally observed:

- average levitation (AL) - performances are similar with performances obtained from three control logics (Section II),

- stabilized levitation (SL) - one position levitation performances were improved (obtained in [15]),

- no levitation state (NLS) - levitation of the ball was not performed,

- levitation with motions (LWM) - two position levitation was obtained.

Results from Table I showed that the activation function based on orthogonal polynomials positively affects the realization of improved two position ball levitation.

Magnetic levitation system used for experimental purposes is susceptible to external conditions and environmental impacts. Minor deviations occurred in accordance to these imperfections during experiments repetition. Hence, two position levitation of the ball is provided by using the function

$$
\begin{gathered}
P_{4}^{(1, \delta)}(x)=a_{44}^{(1, \delta)} x^{4}+a_{43}^{(1, \delta)} x^{3}+ \\
+a_{42}^{(1, \delta)} x^{2}+a_{41}^{(1, \delta)} x+a_{40}^{(1, \delta)},
\end{gathered}
$$

where:

$$
\left\{\begin{array}{l}
a_{44}^{(1, \delta)}=-\frac{(\delta+2)(\delta+4)(3 \delta+4)}{12}, \\
a_{43}^{(1, \delta)}=\frac{(\delta+1)(\delta+3)(2 \delta+3)}{2}, \\
a_{42}^{(1, \delta)}=-\frac{(\delta+1)(\delta+2)(3 \delta+2)}{2}, \\
a_{41}^{(1, \delta)}=\frac{(\delta+1)(2 \delta+1)(3 \delta+4)}{6}, \\
a_{40}^{(1, \delta)}=\frac{\delta^{3}}{4} .
\end{array}\right.
$$

The function $P_{4}^{(1, \delta)}(x)$ provides improved two position levitation of the metallic ball for all examined function values. It is shown experimentally that the most stable results were obtained for selecting parameter $\delta$ in the interval $1.000-1.020$. Range of parameter $\delta$ between $1.000-1.020$ was examined further by performing new experiments on the function $P_{4}^{(1, \delta)}(x)$. Parameter $\delta$ was selected from the specified range with a step size 0.002 .

The best performances of presented orthogonal functions are obtained when the uncertain quantity parameter is selected to be $\delta=1.02$. The function $P_{4}^{(1, \delta)}(x)$ then takes the following form

$$
\begin{aligned}
& P_{4}^{(1, \delta)}(x)=-8,9194 x^{4}+20,4634 x^{3}- \\
& -15,4340 x^{2}+7,2257 x-0,2653 .
\end{aligned}
$$

The main experiment is performed after the new-formed activation function (14) is implemented in the neural network. 
TABLE I. MAGNETIC LEVITATION SYSTEM EXPERIMENTS BASED ON NEURAL NETWORK ACTIVATION FUNCTION (PARAMETER $\Delta$ ).

\begin{tabular}{|c|c|c|c|c|c|c|c|c|c|c|c|}
\hline & \multicolumn{11}{|c|}{ Parameter $\delta$} \\
\hline Function & 1.000 & 1.005 & 1.010 & 1.015 & 1.020 & 1.025 & 1.030 & 1.035 & 1.040 & 1.045 & 1.050 \\
\hline$P_{1}^{(1, \delta)}(x)$ & \multicolumn{6}{|c|}{$\mathrm{SL}$} & \multicolumn{5}{|c|}{$\mathrm{AL}$} \\
\hline$P_{2}^{(1, \delta)}(x)$ & \multicolumn{11}{|c|}{$\mathrm{AL}$} \\
\hline$P_{3}^{(1, \delta)}(x)$ & \multicolumn{7}{|c|}{$\mathrm{AL}$} & \multicolumn{4}{|c|}{ NLS } \\
\hline$P_{4}^{(1, \delta)}(x)$ & \multicolumn{11}{|c|}{ LWM } \\
\hline
\end{tabular}

System performances are presented in Fig. 5 and Fig. 6. Figure 5 shows the results of developed neural network when specified amplitude is $10^{-4} \mathrm{~m}$. A new response is compared with responses from Fig. 3.

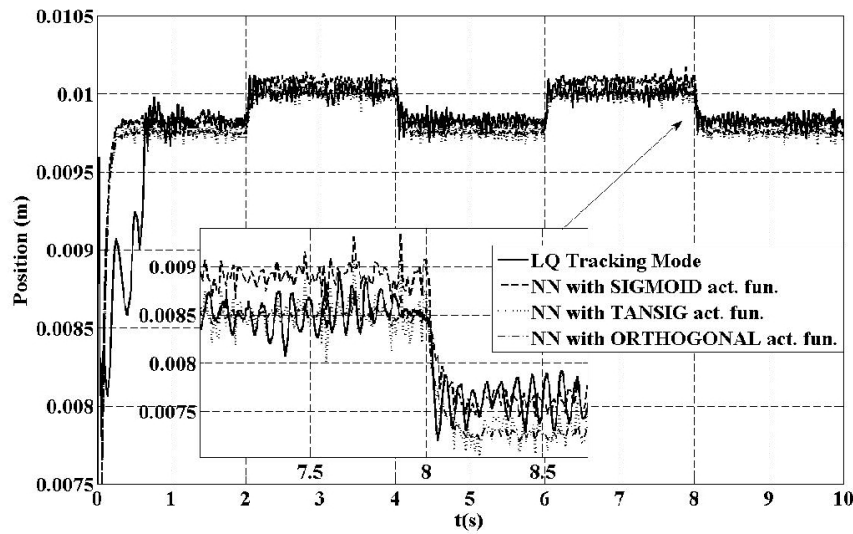

Fig. 5. Two-position ball movement comparison between Fig. 3 and developed network after $4^{\text {th }}$ order orthogonal activation function implementation, amplitude $A_{1}=10^{-4}$.

It can be concluded that the new network based on orthogonal activation function provides similar results compared to control logics presented in Section II. This activation function showed stable levitation performances for each repeated experiment. Figure 6 presents a comparison of experimental results when specified amplitude is $A_{2}=10^{-3} \mathrm{~m}$.

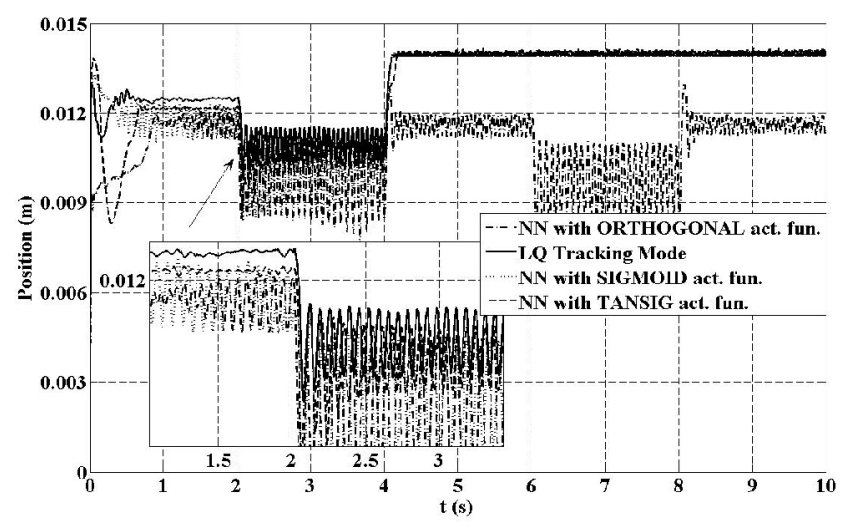

Fig. 6. Two-position ball movement comparison between Fig. 4 and developed network after $4^{\text {th }}$ order orthogonal activation function implementation, amplitude: $A_{2}=10^{-3} \mathrm{~m}$.

It can be easily concluded that the new network provided stable levitation when the amplitude is increased, which is a significant performance improvement. Amplitude value $A_{2}$ is confirmation that properly used neural network with developed orthogonal activation function can improve levitation performances and resolve two position levitation problem from Section II (Fig. 4).

A large oscillations appearance is the main observed disadvantage. Oscillations are up to $12,8 \%$ compared to the ideal lower levitation position, which is not a satisfactory result. Oscillations are further $2,5 \%$ on the higher position, which is acceptable. Default system oscillations are $1 \%$ (Fig. 3) and compared to the new levitation performances (Fig. 5), the system lost on precision. Those disadvantages will be examined in a future work.

\section{CONCLUSIONS}

The magnetic levitation problem is examined in the paper. Two position ball levitation was performed by three control structures: default LQ tracking mode, and two modified LQ structures with implemented NARX neural networks. All examined control logics did not obtain satisfactory results when the specified amplitude between the two positions was greater than $10^{-4} \mathrm{~m}$. This problem was further analysed and resolving method is proposed. Neural network with orthogonal polynomial activation function is presented and integrated into the standard LQ control logic. Generalized quasi-orthogonal polynomials from $1^{\text {st }}$ to $4^{\text {th }}$ order are used for experimental purposes. Improved two position levitation is observed in the instances when $4^{\text {th }}$ order activation functions are used. Function coefficients are adjusted by properly selecting uncertain quantity parameter $\delta$. Experimental results are shown and it can be concluded that amplitude performances improved significantly during two position levitation process. New maximal obtained movement of the metal ball between levitation positions (the amplitude) is $10^{-3} \mathrm{~m}$ approximately. Developed activation function influenced the appearance of larger levitation oscillations compared to the standard control logic performances. Finally, modified control logic proved to be an effective method for significant improvement of magnetic levitation performances.

\section{REFERENCES}

[1] J. Jeon, M. Caraiani, K. Lee, D. Hwang, J. Lee, Y. Kim, T. Nam, S. Kim, "High-precision control of magnetic levitation system", in Proc. 31st Annual Conf. Industrial Electronics Society, GyeonggiDo, Korea, 2005, pp. 1-6. [Online]. Available: http://dx.doi.org/ 10.1109/IECON.2005.1569313

[2] H.-K. Chiang, C.-C. Fang, W.-B. Lin, G.-W. Chen, "Second-order sliding mode control for a magnetic levitation system", in Proc. 8th Asian Control Conf., Kaohsiung, Taiwan, 2011, pp. 602-607. 
[3] W. Barie, J. Chiasson, "Linear and nonlinear state-space controllers for magnetic levitation”, International Journal of Systems Science, vol. 27, no. 11, pp. 1153-1163, 1996. [Online]. Available: http://dx.doi.org/10.1080/00207729608929322

[4] K. S. Peterson, J. W. Grizzle, A. G. Stefanopoulou, "Nonlinear control for magnetic levitation of automotive engine valves", IEEE Trans. Control Systems Technology, vol. 14, no. 2, pp. 346-354, 2006. [Online]. Available: http://dx.doi.org/10.1109/TCST.2005. 863669

[5] R. Morales-Herrera, H. Sira-Ramirez, "Trajectory tracking for the magnetic ball levitation system via exact feedforward linearization and GPI control", International Journal of Control, vol. 83, no. 6, pp. 1155-1166, 2010. [Online]. Available: http://dx.doi.org/10.1080 00207171003642196

[6] T. H. Luat, J.-H. Cho, Y.-T. Kim, "Fuzzy-tuning PID controller for nonlinear electromagnetic levitation system", in: Soft Computing in Intelligent Control, S. Kim, J.-W. Jung and N. Kubota, Eds., Advances in Intelligent Systems and Computing, Springer International Publishing, vol. 272, pp. 17-28, 2014. [Online]. Available: http://dx.doi.org/10.1007/978-3-319-05570-1_3

[7] C.-A. Dragos, R.-E. Precup, E. M. Petriu, M. L. Tomescu, St. Preitl, R.-C. David, M.-B. Radac, "2-DOF PI-fuzzy controllers for a magnetic levitation system", in Proc. of 8th Int. Conf. Informatics in Control, Automation and Robotics, Noordwijkerhout, Netherlands, vol. 1, 2011, pp. 111-116. [Online]. Available: http://dx.doi.org/ $10.5220 / 0003537201110116$

[8] K. Ishaque, Y. Saleem, S. S Abdullah, M. Amjad, M. Rashid, S. Kazi, "Modeling and control of magnetic levitation system via fuzzy logic controller", in Proc. 4th Int. Conf. Modeling, Simulation and Applied Optimization, Kuala Lumpur, Malaysia, 2011, pp. 1-6. [Online]. Available: http://dx.doi.org/10.1109/ICMSAO.2011. 5775532

[9] R.-E. Precup, C.-A. Bojan-Dragos, E. M. Petriu, M.-B. Radac, A.-I. Stinean, "Results on optimal tuning of fuzzy models of magnetic levitation systems", International Journal of Artificial Intelligence, vol. 13 , no. 2 , pp. 57-72, 2015 .

[10] U. Farooq, J. Gu, M. E. El-Hawary, G. Abbas, M. U. Asad, “An interval type-2 fuzzy regulator for magnetic levitation system", in Proc. IEEE 28th Canadian Conf. Electrical and Computer Engineering, Halifax, NS, Canada, 2015, pp. 424-431. [Online] Available: http://dx.doi.org/10.1109/CCECE.2015.7129315

[11] C.-A. Bojan-Dragos, A.-I. Stinean, R.-E. Precup, S. Preitl, E. M. Petriu, "Model predictive control solution for magnetic levitation systems", in Proc. 20th Int. Conf. Methods and Models in Automation and Robotics, Miedzyzdroje, Poland, 2015, pp. 139-144. [Online]. Available: http://dx.doi.org/10.1109/MMAR.2015.7283861

[12] S. Sgaverdea, C.-A. Bojan-Dragos, R.-E. Precup, S. Preitl, A.-I Stinean, "Model predictive controllers for magnetic levitation systems", in Proc. IEEE 10th Jubilee Int. Symposium on Applied Computational Intelligence and Informatics, Timisoara, Romania, 2015, pp. 171-176. [Online]. Available: http://dx.doi.org/10.1109/ SACI.2015.7208193

[13] Inteco, The Magnetic Levitation System (MLS)-User's Manual. Online. [Available]: www.inteco.com.pl

[14] M. Lairi, G. Bloch, "A neural network with minimal structure for maglev system modeling and control", in Proc. IEEE International Intelligent Control/Intelligent Systems and Semiotics, Cambridge, USA, 1999, pp. 40-45. [Online]. Available: http://dx.doi.org/ 10.1109/ISIC.1999.796627

[15] M. Saberi, H. Altafi, S. Alizadeh, "Control of the magnetic suspension system with a three-degree-of-freedom using RBF neural network controller", International Journal of Computer and
Electrical Engineering, vol. 4, no. 2, pp. 121-126, 2012. [Online]. Available: https://doi.org/10.7763/IJCEE.2012.V4.462

[16] P. S. Shiakolas, S. R. Schenck, D. Piyabongkarn, I. Frangeskou, "Magnetic levitation hardware-in-the-loop and MATLAB-based experiments for reinforcement of neural network control concepts", IEEE Trans. Education, vol. 47, no. 1, pp. 33-41, 2004. [Online]. Available: http://dx.doi.org/10.1109/TE.2003.817616

[17] P. Haley, D. Soloway, B. Gold, "Real-time adaptive control using neural generalized predictive control", in Proc. American Control Conf., San Diego, USA, 1999, vol. 6, pp. 4278-4282. [Online]. Available: http://dx.doi.org/10.1109/ACC.1999.786371

[18] Y. Qina, H. Penga, F. Zhoua, X. Zenga, J. Wu, "Nonlinear modeling and control approach to magnetic levitation ball system using functional weight RBF network-based state-dependent ARX model", Journal of the Franklin Institute, vol. 352, no. 10, pp. 4309-4338, 2015. [Online] Available: http://dx.doi.org/10.1016 /j.jfranklin.2015.06.014

[19] D. Antic, M. Milovanovic, S. Nikolic, M. Milojkovic, S. Peric, "Simulation model of magnetic levitation based on NARX neural networks", International Journal of Intelligent Systems and Applications, vol. 5, no. 5, pp. 25-32, 2013. [Online] Available: http://dx.doi.org/10.5815/ijisa.2013.05.04

[20] S. S. Nikolic, D. S. Antic, M. T. Milojkovic, M. B. Milovanovic, S. Lj. Peric, D. B. Mitic, "Application of neural networks with orthogonal activation functions in control of dynamical systems", International Journal of Electronics, vol. 103, no. 4, pp. 667-685 [Online]. Available: http://dx.doi.org/10.1080/00207217.2015. 1036811

[21] I. Sahin, I. Koyuncu, "Design and implementation of neural networks neurons with RadBas, LogSig, and TanSig activation functions on FPGA", Elektronika ir Elektrotechnika, vol. 120, no. 4, pp. 51-54, 2012. [Online]. Available: http://dx.doi.org/10.5755/j01.eee.120.4 1452

[22] W. Zou, Y. Zhu, L. Cai, "Fourier-neural-network-based learning control for a class of nonlinear systems with flexible components", IEEE Trans. Neural Networks, vol. 20, no. 1, pp. 139-151, 2009. [Online]. Available: http://dx.doi.org/ 10.1109/TNN.2008.2006496

[23] S. S. Nikolic, D. S. Antic, S. Lj. Peric, N. B. Dankovic, M. T. Milojkovic, "Design of generalised orthogonal filters: Application to the modelling of dynamical systems", International Journal of Electronics, vol. 103, no. 2, pp. 269-280. [Online]. Available: http://dx.doi.org/10.1080/00207217.2015.1036367

[24] M. T. Milojkovic, D. S. Antic, S. S. Nikolic, Z. D. Jovanovic, S. Lj. Peric, "On a new class of quasi-orthogonal filters", International Journal of Electronics, vol. 100, no. 10, pp. 1361-1372, 2013. [Online]. Available: http://dx.doi.org/10.1080/00207217.2012. 743087

[25] B. Dankovic, S. Nikolic, M. Milojkovic, Z. Jovanovic, "A class of almost orthogonal filters", Journal of Circuits, Systems, and Computer, vol. 18, no. 5, pp. 923-931, 2009. [Online]. Available: http://dx.doi.org/10.1142/S0218126609005447

[26] M. Milojkovic, S. Nikolic, B. Dankovic, D. Antic, Z. Jovanovic, "Modelling of dynamical systems based on almost orthogonal polynomials", Mathematical and Computer Modelling of Dynamical Systems, vol. 16, no. 2, pp. 133-144, 2010. [Online]. Available: http://dx.doi.org/10.1080/13873951003740082

[27] D. Antic, B. Dankovic, S. Nikolic, M. Milojkovic, Z. Jovanovic, "Approximation based on orthogonal and almost orthogonal functions", Journal of the Franklin Institute, vol. 349, no. 1 , pp. 323-336, 2012. [Online]. Available: http://dx.doi.org/10.1016/ j.jfranklin.2011.11.006 